\title{
Trattamento e produzione di energia dalle acque reflue
}

\author{
Vincenzo Marino ${ }^{1}$ \\ ${ }^{1}$ University of Salerno
}

\begin{abstract}
In un mondo in cui il consumo di risorse è sempre più insostenibile, utilizzare energia da fonti rinnovabili è diventata una delle principali sfide dell'uomo. Allo stesso tempo, il consumo di energia rinnovabile comporta la riduzione di emissioni di inquinanti. Le celle a combustibile microbiche (MFC) offrono nuove opportunità per la produzione sostenibile di energia dalle acque reflue. Il sistema, infatti, utilizzato a valle dell'ormai consolidato processo di trattamento con Bioreattori a Membrana (MBR), si presta come processo per la depurazione delle acque reflue e la contemporanea produzione energetica, dal momento che i sottoprodotti della cella a combustibile microbica sono acqua reflua depurata ed elettricità. Tuttavia, l'impiego di MFC è ancora limitato a causa delle incognite che presentano alcuni parametri delle fasi di processo. I risultati futuri potrebbero essere migliori in quanto non sono stati ancora studiati alcuni aspetti che potrebbero influenzare significativamente l'efficienza del processo.
\end{abstract}

\section{Introduzione}

Le problematiche connesse all'esaurimento di combustibili fossili e al riscaldamento globale si sono ingigantite negli ultimi anni. Pertanto, l'attenzione dell'uomo si è spostata verso soluzioni che andassero a migliorare la sostenibilità energetica e ambientale.

A tal proposito, si è palesata la necessità di sviluppare schemi di processo che ottimizzassero l'efficienza di un determinato sistema e che, al tempo stesso, offrissero la possibilità di recuperare energia.

Tali considerazioni assumono un'importante rilevanza in molti campi dell'ingegneria, tra i quali il trattamento delle acque reflue.

Tra i diversi processi di depurazione delle acque reflue, uno dei più applicati prevede l'impiego di bioreattori a membrana (MBR). Tuttavia, il 


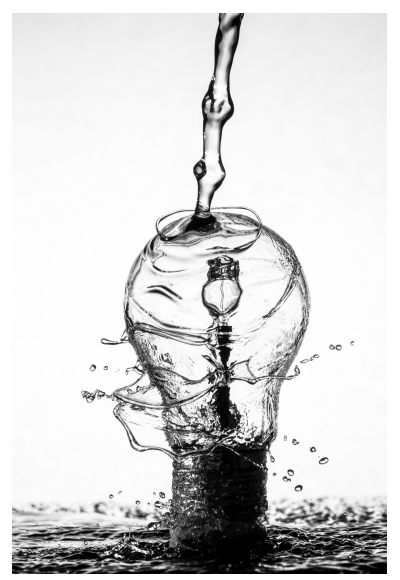

Figure 1: Le acque reflue come fonte per il recupero di energia

fouling di membrana è ancora un grosso ostacolo che limita una più rapida commercializzazione di MBR ${ }^{1}$.

Pertanto, i sistemi MBR sono stati combinati con altre tecnologie per estenderne l'efficacia nel trattamento di una vasta gamma di contaminanti e per migliorare il controllo delle incrostazioni ${ }^{2}$.

Esistono differenti tecnologie che vengono utilizzate ad integrazione degli impianti MBR, le quali mirano a migliorare le prestazioni del trattamento. Tra le altre, negli ultimi anni si sta diffondendo l'utilizzo di celle a combustibile microbiche; infatti come evidenziato da ${ }^{3}$, i sistemi bioelettrochimici (BES), come le celle a combustibile microbiche (MFC), rappresentano un approccio promettente per il trattamento simultaneo delle acque reflue mentre si genera elettricità.

Gli studi a sostegno di questa tesi sono molteplici: in un precedente studio, ${ }^{4}$ hanno osservato che l'integrazione dei processi elettrochimici in un MBR ha il vantaggio di migliorare le prestazioni del trattamento soprattutto in termini di rimozione dei nutrienti, oltre ai precursori del fouling convenzionale come le sostanze polimeriche extracellulari legate (bEPS) e i prodotti microbici solubili (SMP).

Secondo ${ }^{5}$ I'MFC è in grado di convertire l'energia chimica nel substrato organico direttamente in energia elettrica utile dalla reazione catalitica dei microrganismi e al tempo stesso far si che i fanghi vengano idrolizzati, convertiti e ridotti durante la produzione di elettricità.

Infine, ${ }^{6}$ hanno raggiunto una riduzione del $25,1 \%$ dei solidi sospesi totali (TSS) e una riduzione del $22,8 \%$ dei solidi sospesi volatili (VSS) in un MFC che utilizzava fanghi di depurazione come combustibile.

Nonostante ciò, la commercializzazione di MFC è limitata a causa della 
bassa produzione di energia e dei suoi costi elevati. Esistono, dunque, diverse sfide da superare per sviluppare le prestazioni e la commercializzazione degli MFC. 7 .

\section{Analisi delle prestazioni di un MFC applicato a valle di un MBR}

Lo studio analizzato si è incentrato sull'analisi e valutazione di alcuni parametri che possono influenzare in maniera significativa le prestazioni di un MFC integrato ad un MBR. L'influenza di differenti concentrazioni di TSS all'interno dei fanghi di alimentazione è uno di questi; ad esso si aggiungono gli effetti che un processo bioeletettrochimico quale I'MFC ha sulla degradazione della sostanza organica, sulla riduzione dei fanghi e sul fouling di membrana.

Le prove di laboratorio sono state effettuate mediante un reattore contenente una miscela e fanghi attivati aerobici da un MBR pilota per il trattamento delle acque reflue, alimentato in batch per quattro cicli caratterizzati da differente contenuto di TSS e diversa durata. Analizzando i campioni prelevati ad inizio e fine di ogni ciclo di alimentazione, è stato possibile ricavare informazioni sulle proprietà dei fanghi ridotti e sulle caratteristiche dei processi di riduzione. Inoltre, mediante l'utilizzo di strumenti elettrochimichi è stato studiato il diretto collegamento tra la rimozione di substrato organico e la produzione di energia.

Dopo aver stabilizzato il biofilm anodico elettroattivo, sono stati effettuati test sul sistema con l'obiettivo di distinguere le attività bioelettrochimiche da quelle non bioelettrochimiche e confrontarle. Nell'MFC sono stati riscontrati tassi di rimozione del tCOD più alti del $30 \%$ rispetto all'MFC a circuito aperto. E' stata ottenuta, inoltre, una riduzione dei VSS di circa il $43 \%$ con una velocità di rimozione massima pari a $150 \mathrm{mg} \mathrm{L}-1 \mathrm{~d}-1$.

La generazione di elettricità è avvenuta raggiungendo una densità di corrente massima di 2,0 A m-2 in corrispondenza del quarto ciclo caratterizzato da una più alta presenza di fanghi: I'aumento della concentrazione dei fanghi ha permesso ai batteri di assorbire maggiore quantità di COD e quindi degradare più sostanza organica producendo un quantitativo maggiore di elettricità.

Per quanto riguarda l'incrostazione di membrana è stata studiata l'idrofobicità e il rapporto SMPp/SMPc che regola la reversibilità delle incrostazioni che si formano ed è considerato la maggiore causa di incrostazione di membrana un un $\mathrm{MBR}^{8}$; dopo il trattamento in MFC, è aumentata sia l'idrofobicità dei fanghi di circa il 50\% sia il rapporto SMPp/SMPc. Quest'ultimo aspetto incide in maniera significativa sull'efficienza del processo; infatti, come evidenziato anche da ${ }^{9}$, rispetto a un MBR convenzionale, uno degli effetti significativi dovuti all'integrazione dell'MFC sulle 
proprietà dei fanghi è l'aumento del rapporto $\mathrm{SMPp} / \mathrm{SMPc}$ che porta alla mitigazione del fouling di membrana.

\section{Conclusioni}

Lo studio ha dimostrato come l'integrazione di un sistema MFC a un impianto MBR migliori l'efficienza di riduzione dei fanghi, come è evidenziato dai risultati ottenuti in termini di rimozione del tCOD e dei VSS. Tali risultati hanno anche mostrato una ottimizzazione delle prestazioni nel secondo ciclo di alimentazione, da cui si evince che il degrado della sostanza organica da parte dei batteri è favorita a un contenuto limitato di fanghi.

Lo stesso discorso non vale per la produzione di elettricità perché, anche se in valori modesti, essa è aumentata con l'aumento della concentrazione di fanghi.

Infine, sono stati notati miglioramenti nel contrastare il fenomeno di fouling di membrana grazie all'aumento dell'idrofobicità dei fanghi che diminuisce la propensione a generare incrostazioni di membrana, e del rapporto $\mathrm{SMPp} / \mathrm{SMPc}$ che permette di conferire alle incrostazioni che si formano una maggiore irreversibilità.

\section{References}

1.M.Yao, B.Ladewig \& K.Zhang. Identification of the change of soluble microbial products on membrane fouling in membrane bioreactor (MBR). Desalination 278, 126-131 (2011).

2.L.Borea et al.. Are pharmaceuticals removal and membrane fouling in electromembrane bioreactor affected by current density?. Science of The Total Environment 692, 732-740 (2019).

3.L.Borea et al.. Microbial fuel cell technology as a downstream process of a membrane bioreactor for sludge reduction. Chemical Engineering Journal 326, 222-230 (2017).

4.L.Borea, V.Naddeo \& V.Belgiorno. Application of electrochemical processes to membrane bioreactors for improving nutrient removal and fouling control. Environmental Science and Pollution Research 24, 321-333 (2016).

5.B.Xiao, F.Yang \& J.Liu. Enhancing simultaneous electricity production and reduction of sewage sludge in two-chamber MFC by aerobic sludge digestion and sludge pretreatments. Journal of Hazardous Materials $\mathbf{1 8 9}$, 444-449 (2011). 
6.X.Su, Y.Tian, Z.Sun, Y.Lu \& Z.Li. Performance of a combined system of microbial fuel cell and membrane bioreactor: Wastewater treatment sludge reduction, energy recovery and membrane fouling. Biosensors and Bioelectronics 49, 92-98 (2013).

7.P.Choudhury, U.S.P.Uday, T.K.Bandyopadhyay, R.N.Ray \& B.Bhunia. Performance improvement of microbial fuel cell (MFC) using suitable electrode and Bioengineered organisms: A review. Bioengineered 8, 471-487 (2017).

8.A.Drews, M.Vocks, U.Bracklow, V.Iversen \& M.Kraume. Does fouling in MBRs depend on SMP?. Desalination 231, 141-149 (2008).

9.G.Zhou et al.. Assessment of a novel overflow-type electrochemical membrane bioreactor (EMBR) for wastewater treatment energy recovery and membrane fouling mitigation. Bioresource Technology 196, 648-655 (2015). 